\title{
Multi-frequency scatter-broadening evolution of pulsars
}

\author{
M. A. Krishnakumar ${ }^{1,2,3}$, Bhal Chandra Joshi ${ }^{2}$ and P. K. \\ Manoharan ${ }^{1,2}$ \\ ${ }^{1}$ Radio Astronomy Centre, NCRA-TIFR, Ooty - 643 001, India \\ ${ }^{2}$ National Cente for Radio Astrophysics, TIFR, Pune - 411007 \\ ${ }^{3}$ Bharathiar University, Coimbatore - 641046 \\ email: kkma@ncra.tifr.res.in
}

\begin{abstract}
In this paper, we present our study on multi-frequency scatter-broadening observations of a large sample of pulsars, made using the Ooty Radio Telescope (ORT) and the Giant Metrewave Radio Telescope (GMRT). For each pulsar, the scatter-broadening time scales $\left(\tau_{s c}\right)$ have been estimated at different observing frequencies and the dependence of $\tau_{s c}$ with the observing frequency, i.e., the frequency scaling index $(\alpha)$ has been obtained. We report estimates of $\alpha$ for a set of 39 pulsars, of which 31 are completely new and provide the first-time measurement on about $50 \%$ of the sample. This enhanced sample suggests that almost $65 \%$ of the pulsars have an $\alpha$ much lower than the conventional value of 4.4 for a Kolmogorov type turbulence spectrum, and a considerably large scattering strength. An increase in scattering strength is observed with the distance to the pulsar in the Galaxy.
\end{abstract}

Keywords. ISM: general - pulsars: general - scattering

\section{Introduction}

The investigation of the turbulence characteristics of the ionized Inter-stellar medium (IISM) along lines of sight to pulsars is important for an understanding of the IISM as well as for disentangling the effects of IISM in the detection of Gravitational Waves. The IISM affects the pulsar signal in different ways, viz., cold plasma dispersion, scintillation, scattering, etc. Although more than 2,500 pulsars are discovered till date, the measurements of pulse scatter-broadening $\left(\tau_{s c}\right)$ at multiple frequencies, and consequently the estimation of the frequency scaling index of scattering, $\alpha$, are only available for $\sim 60$ pulsars. An enhancement of this sample is essential in understanding the turbulence characteristics in different regions of the Galaxy and to study the effects of HII regions, supernova remnants, etc. on scattering. In this study, we concentrate on measurements of $\tau_{s c}$ at multiple frequencies for a large sample of pulsars to understand the turbulence characteristics in their lines of sight.

\section{Observations and Analysis}

We observed 44 pulsars using the Giant Metrewave Radio Telescope, Pune, at frequencies 150, 235 and $610 \mathrm{MHz}$ and the Ooty Radio Telescope (ORT) at $327 \mathrm{MHz}$. We used archival data from the Long Wavelength Array (LWA1), in New Mexico, between frequencies $30-90 \mathrm{MHz}$ for three pulsars. The data were reduced to profiles at each of the observing frequencies for further analysis. The LWA1 data were sub-banded into 8 sub-band profiles and these profiles were used for further analysis. For more details regarding the observations and data reduction, please see Krishnakumar et al. (2017). 


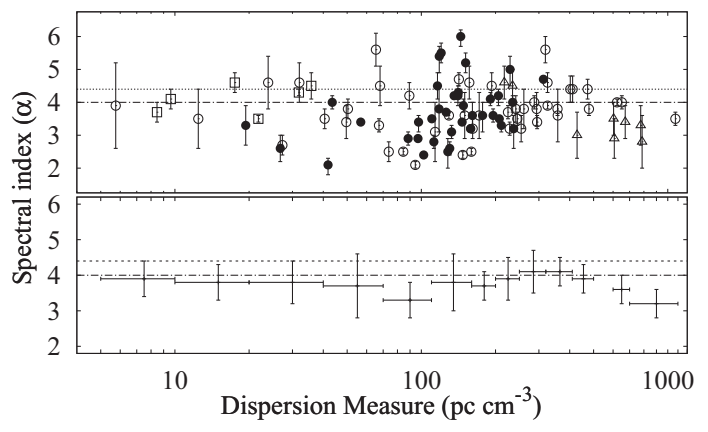

Figure 1. The distribution of $\alpha$ as a function of DM is plotted. Top panel shows the measurements from Lewandowski et al. (2015) (open circles); Löhmer et al. (2001) (triangles); Cordes et al. (1985) (squares) and our measurements (filled circles). In the bottom panel, the average of $\alpha$ in $13 \mathrm{DM}$ bins is shown.

To estimate $\tau_{s c}$, we used the method as detailed in Krishnakumar et al. (2017). We used an unscattered high frequency profile template to convolve with the observed scatterbroadened profile to measure $\tau_{s c}$. These measurements of $\tau_{s c}$ at different frequencies were used to estimate, $\alpha$ by passing it through a Markov-Chain-Monte-Carlo method and the median of this distribution is taken as $\alpha$.

\section{Results and Discussions}

We report $\alpha$ measurements for a sample of 39 pulsars, with 31 new measurements, thus enhancing the current sample by about $50 \%$. The distribution of $\alpha$ for 99 pulsars as a function of dispersion measure (DM) is given in Figure 1. We see a peculiar dip in $\alpha$ at a DM of $\sim 100 \mathrm{pc} \mathrm{cm}^{-3}$. This might be due to a small number of pulsars in this DM bin and motivate further observations of a large set of pulsars in this DM range to confirm this. We also see a general trend of increase in the scattering strength, $C_{n_{e}}^{2}$ at larger distances, suggesting that the inhomogeneities in the line of sight increases with distance. DM independent distance estimates are required to understand this in a much better way, since most of the distance estimates are model dependent. We see that, for the pulsars with $\alpha$ shallower than 4.4, the scattering strength, $C_{n_{e}}^{2}$, is high. About $65 \%$ of the pulsars in our sample show an $\alpha$ flatter than 4.4 and large value of $C_{n_{e}}^{2}$, indicating that these lines of sight favour a non-Kolmogorov type turbulence characteristics. It also suggest that there may be multiple-scatterers in the line of sight, which result in such an effect.

Although we have increased the current sample of $\alpha$ measurements to $\sim 100$ pulsars with this study, one requires a large sample of such measurements to clearly understand the turbulence characteristics in the IISM. We have made more $\alpha$ measurements by observing with telescopes across the globe at different frequency - DM combinations, and also by analysing the archival datasets. The results from this effort will double the current sample of $\alpha$ measurements and will give insights into the turbulence in the IISM in a much better way than what we know currently.

\section{References}

Cordes, J. M., Weisberg, J. M., \& Boriakoff, V., 1985, ApJ, 288, 221

Krishnakumar, M. A., Joshi, Bhal Chandra \& Manoharan, P. K., 2017, ApJ, 846, 104

Lewandowski, W., Roźko, K., Kijak, J., Bhattacharyya, B., \& Roy, J., 2015, MNRAS, 454, 2517

Löhmer, O., Kramer, M., Mitra, D., Lorimer, D. R., \& Lyne, A. G., 2001, ApJ, 562, 157 